\title{
AN INTEGRATED FLEXIBLE SELF-CALIBRATION APPROACH FOR 2D LASER SCANNING RANGE FINDERS APPLIED TO THE HOKUYO UTM-30LX-EW
}

\author{
David Mader, Patrick Westfeld, Hans-Gerd Maas
}

Institute of Photogrammetry and Remote Sensing, Technische Universität Dresden, Germany

\{david.mader, patrick.westfeld, hans-gerd.maas\}@tu-dresden.de

Commission V

KEY WORDS: LIDAR, laser range finder, terrestrial laser scanning, error model, calibration, bundle adjustment, variance component estimation

\begin{abstract}
:
The paper presents a flexible approach for the geometric calibration of a 2D infrared laser scanning range finder. It does not require spatial object data, thus avoiding the time-consuming determination of reference distances or coordinates with superior accuracy. The core contribution is the development of an integrated bundle adjustment, based on the flexible principle of a self-calibration. This method facilitates the precise definition of the geometry of the scanning device, including the estimation of range-measurement-specific correction parameters. The integrated calibration routine jointly adjusts distance and angular data from the laser scanning range finder as well as image data from a supporting DSLR camera, and automatically estimates optimum observation weights. The validation process carried out using a Hokuyo UTM-30LX-EW confirms the correctness of the proposed functional and stochastic contexts and allows detailed accuracy analyses. The level of accuracy of the observations is computed by variance component estimation. For the Hokuyo scanner, we obtained $0.2 \%$ of the measured distance in range measurement and 0.2 deg for the angle precision. The RMS error of a 3D coordinate after the calibration becomes $5 \mathrm{~mm}$ in lateral and $9 \mathrm{~mm}$ in depth direction. Particular challenges have arisen due to a very large elliptical laser beam cross-section of the scanning device used.
\end{abstract}

\section{INTRODUCTION}

2D laser scanners based on the time-of-flight principle consume less power, are compact and light-weight as well as reasonably priced. Installed on a moving platform, such a measurement device represents an interesting alternative to stereo vision techniques or 3D laser scanner systems. It thus becomes an indispensable instrument for localization, mapping and obstacle detection in land or airborne mobile robotic applications.

Rogers III et al. (2010) use compact laser scanning range finders (LSRF) for simultaneous mobile robot localization and mapping (SLAM) in an indoor office environment. In (Krüger et al., 2013; Nowak et al., 2013), single-layer laser scanner data are used to detect and localize unmanned swarm vehicles for an extra-terrestrial exploration mission. Serranoa et al. (2014) present a navigation algorithm based on 2D laser scanner data to allow seamless inand outdoor navigation of an unmanned aerial vehicles (UAVs). In (Scherer et al., 2012), a LSRF is fixed on a flying robot to perform autonomous river mapping. In (Kuhnert and Kuhnert, 2013), a small LSRF is attached to a micro drone for precise 3D power-line monitoring. (Djuricic and Jutzi, 2013) support a UAV by multiple-pulse laser scanning devices and overcome the limitations in form of low visibility due to soft obstacles like fog or rain. Holz et al. (2013) take advantage of high LSRF scan rates and propose a method for obstacle avoidance that allows fully autonomous UAV flights.

Whether for navigation or for mapping tasks, a calibration of the laser scanning system is reasonable to maximize data accuracy. This contribution proposes a flexible method for LSRF system self-calibration. It is structured as follows: The functional principle and the ideal geometric measurement model for a singlelayer LSRF are introduced in section 2. In section 3, the error characteristic of a light-weight 2D laser scanner is described, error sources are identified and different calibration strategies for correction are discussed. The integrated self-calibrating bundle adjustment approach presented here and the results achieved are described in detail in sections 4 and 5. Finally, the work is summarized and an outlook is given in section 6 .

\section{SENSOR}

Laser scanners are active sensors, emitting near infrared light, which is backscattered from the object surface to the sensor. The distance is measured by timing the round-trip path of the laser beam (time-of-flight) or by measuring the phase difference between the emitted and the received signals (phase-shift). The intensity of the reflected light is often additionally stored as measure for surface's albedo. A comprehensive overview of laser scanning technology is given in (Vosselman and Maas, 2010).

A single-layer LSRF is typically realized as time-of-flight ranging system. It uses pulsed laser light beams to directly measure a time delay created by light traveling from the sensor to the object and back. The laser scanning unit measures very fast by sending light beams into the center of a continuously rotating mirror. Latest devices like the 210 gram light-weight Hokuyo UTM30LX-EW (figure 1a) shift this mirror with an angular resolution of $0.25 \mathrm{deg}$ between each measurement in a few microseconds. With $270 \mathrm{deg}$ field of view, the Hokuyo scanner provides 1080 measurements per scan line in $25 \mathrm{~ms}$. The scanning range is $0.1 \mathrm{~m}$ to $30 \mathrm{~m}$. Multi-echo functionality allows to receive up to three echoes of a single emitted light pulse.

The spherical coordinates of an object point are given as $(D, \alpha, \beta)$, where $D$ is the measured slant range and $\alpha$ the corresponding horizontal deflection angle. 2D LSRF take measurements over a plane (figure 1b). Consequently, the vertical angle $\beta:=0$ does not exist as a matter of principle. Assuming a righthanded Cartesian coordinate system, the 3D object coordinates 


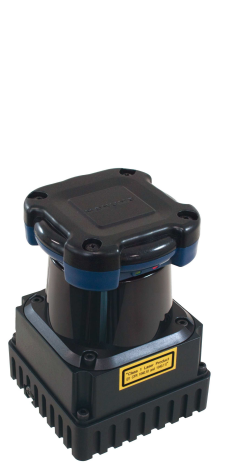

(a)

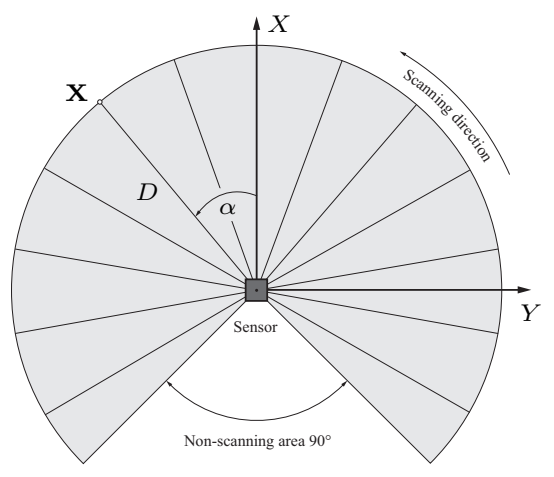

(b)
Figure 1: (a) Laser scanning range finder Hokuyo UTM-30LXEW (Hokuyo, 2014). (b) Measuring principle and coordinate system definition.

become

$$
\mathbf{X}=\left[\begin{array}{l}
X \\
Y \\
Z
\end{array}\right]=D \cdot\left[\begin{array}{c}
\cos \alpha \\
\sin \alpha \\
\sin \beta
\end{array}\right]
$$

Complete 3D scans can be achieved implicitly by moving the platform on which the sensor is mounted. The registration of single measurement stripes can for example be realized by GPS/INS integration (Maier and Kleiner, 2010), by 3D visual SLAM with a camera (Georgiev and Allen, 2004) or by attaining accurate sensor pose with respect to a fixed 3D reference frame (Antone and Friedman, 2007). Considering lever-arm and bore-sight effects finally results in a 3D point cloud representation of the object space. Complete 3D scans can be achieved implicitly by moving the platform on which the sensor is mounted. The registration of single measurement stripes can for example be realized by GPS/INS integration (Maier and Kleiner, 2010), by 3D visual SLAM with a camera (Georgiev and Allen, 2004) or by attaining accurate sensor pose with respect to a fixed 3D reference frame (Antone and Friedman, 2007). Considering lever-arm and boresight effects finally results in a 3D point cloud representation of the object space.

\section{ERROR SOURCES AND CALIBRATION STRATEGIES}

The measured ranges and angles are used for calculating 3D point cloud coordinates (equation 1). The modeling of deviations from the ideal measurement model is required, if accurate 3D information should be delivered. A number of systematic and random errors can act on the original observations of a laser scanner: Effects caused by temperature and running-in behavior or multipath propagation can be decreased or even avoided by an adequate measurement setup. The influence of white noise can be compensated in static, non-time-critical applications by mean of longterm measurements. Laser beam divergence may cause angular displacement errors depending on the location and the shape of the scanned object. Lichti and Gordon (2004) use a probabilistic model to specify the magnitude of this unpredictable error to be equal to one-quarter of the laser beam diameter. Target properties such as color, brightness and material may also have a significant influence on LSRF measurements (Kneip et al., 2009).

The results of the distance and angular measurements are further affected by perturbations - caused for example by imperfections in instrument production - which can be considered in an adequate correction model. Sets of additional parameters for the geometric calibration of terrestrial laser scanning (TLS) instruments are investigated in e. g. (Gielsdorf et al., 2004; Lichti, 2007; Schneider and Schwalbe, 2008), general correction models are summarized in (Vosselman and Maas, 2010). The following considerations base on these works.

A linear distance correction term considers a shift $a_{0}$ of the measurement origin and a scale variation $a_{1}$ caused by counter frequency deviations. A vertical offset of the laser axis from the trunnion axis as well as cyclic distance errors are supposed to be not existent for a 2D single-layer time-of-flight LSRF (section 2). The appropriate distance correction model is defined as

$$
\Delta D=a_{0}+a_{1} \cdot D
$$

The correction model for errors in horizontal direction consists of six correction terms, namely a horizontal encoder (circle) scale error $b_{1}$, two components $b_{2}, b_{3}$ for modeling the horizontal circle eccentricity, two further components $b_{4}, b_{5}$ for modeling the nonorthogonality of the plane containing the horizontal encoder and the rotation axis and, finally, the eccentricity $d_{6}$ of the collimation axis relative to the rotation axis.

$$
\begin{aligned}
\Delta \alpha & =b_{1} \cdot \alpha \\
& +b_{2} \cdot \sin \alpha+b_{3} \cdot \cos \alpha+b_{4} \cdot \sin 2 \alpha+b_{5} \cdot \cos 2 \alpha \\
& +b_{6} \cdot D^{-1}
\end{aligned}
$$

Further additional parameters to correct the collimation and trunnion axis errors are not required due to the 2D scanning principle. Also a correction model for errors in elevation angle is not necessary.

Several calibration strategies are reported in the literature to correct LSRF errors described above. Ye and Borenstein (2002) or Okubo et al. (2009) for example utilize a computer-controller linear motion table for calibrating the scanner device. The experimental configuration is extended in (Kneip et al., 2009) by setting not only reference values for the distance measurement, but for the incidence angle as well. Jain et al. (2011) use time domain techniques for error modeling. Kim and Kim (2011) fit cubic Hermite splines scan-wise to data captured on a translation/rotation stage.

The calibration approaches reviewed above utilize reference stages or comparators for accurate translational and rotational displacement measurements. Reference values can also be provided indirectly by the network geometry determined in the course of a self-calibration, a procedure which reduces time and instrumental effort significantly. Self-calibration strategies are wellestablished for 3D TLS (e. g. Schneider and Maas, 2007; Lichti, 2009). Glennie and Lichti (2010) collect a static data set of planar features in order to determine the internal calibration parameters of a multi-layer LSRF in a bundle adjustment. The further development for compact and light-weight single-layer 2D LSRF is the main core of this contribution.

\section{INTEGRATED SELF-CALIBRATING BUNDLE ADJUSTMENT}

\subsection{Geometric Principle}

The calibration approach should hold the following characteristics: (1) To reduce efforts in time and instrumental resources, the method is based on self-calibration. (2) All necessary types of observations and constraints are integrated in one joint functional 
and stochastic context. (3) The future expansion of the mathematical model developed is facilitated due to its adaptivity and modular implementation. (4) The method requires only a target field, which is stable, compact, portable as well as easy in set-up and dismantle, but it does not require reference values.

The geometric principle is shown in figure 2. It is based on an integrated self-calibrating bundle adjustment of directional distance measurements of a LSRF. Additional images of a digital singlelens reflex camera (DSLR) ensure a stable network geometry.

The reference between LSRF data and DSLR data can either be realized by identifying homologous points in both data sets (which is quite difficult) or by using easily parametrizable geometric primitives (Westfeld and Maas, 2013). In the integrated self-calibrating bundle adjustment presented here, spatial distributed cones functioning as $3 \mathrm{D}$ primitives. Their parameters as well as their poses can be determined from both, LSRF and DSLR observations.

The original LSRF measurements $(D, \alpha)$ are transformed into a local laser scanner coordinate system (lcs) using equation 1 . The 3D object coordinates $\mathbf{X}_{\text {lsrf }}^{\mathrm{lcs}}$ obtained are subsequently transformed into 3D object coordinates $\mathbf{X}_{\mathrm{lsrf}}^{\mathrm{pcs}}$ of a higher-level project coordinate system (pcs):

$$
\mathbf{X}_{\mathrm{lsrf}}^{\mathrm{pcs}}=\mathbf{X}_{\mathbf{0}_{\mathrm{lsrf}}^{\mathrm{pcs}}}^{\mathrm{pcs}}+m_{\mathrm{lcs}}^{\mathrm{pcs}} \cdot \mathbf{R}_{\mathrm{lcs}}^{\mathrm{pcs}} \cdot \mathbf{X}_{\mathrm{lsrf}}^{\mathrm{lcs}}
$$

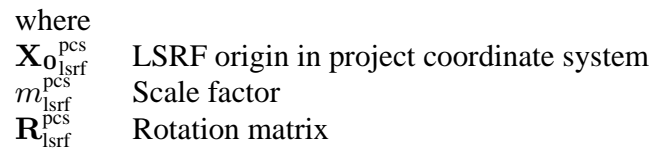

DSLR images of signalized points on target's surfaces are further captured in order to reliably estimate positions and orientations of the cone primitives. The rule for mapping an image point $\mathbf{x}_{\mathrm{cam}}^{\mathrm{scs}}$, which is measured in a local sensor coordinate system (scs), into its corresponding object point $\mathbf{X}_{\text {cam }}^{\mathrm{pcs}}$ is

$$
\mathbf{X}_{\mathrm{cam}}^{\mathrm{pcs}}=\mathbf{X}_{\mathbf{0} \mathrm{cam}}^{\mathrm{pcs}}+m_{\mathrm{scs}}^{\mathrm{pcs}} \cdot \mathbf{R}_{\mathrm{scs}}^{\mathrm{pcs}} \cdot \mathbf{x}_{\mathrm{cam}}^{\mathrm{scs}}
$$

$\begin{array}{ll}\text { where } & \\ \mathbf{X}_{\text {opl ssrf }}^{\text {pcs }} & \text { Camera's projection center } \\ m_{\text {scs }}^{\text {ccs }} & \text { Scale factor } \\ \mathbf{R}_{\text {scs }}^{\text {pcs }} & \text { Rotation matrix }\end{array}$

In order to set the geometric relation between both sensors, all object points $\mathbf{X}_{\text {lsrf,cam }}^{\mathrm{ccs}}$ in a local cone coordinate system (ccs) determined from LSRF resp. DSLR data should satisfy the following general equation of a cone:

$$
0=\frac{r_{\max }^{2}}{h_{\max }^{2}} \cdot\left(h_{\max }-Z_{\mathrm{lsff}, \mathrm{cam}}^{\mathrm{ccs}}\right)^{2}-X_{\mathrm{lsrf}, \mathrm{cam}}^{\mathrm{ccs}^{2}}-Y_{\mathrm{lsrf}, \mathrm{cam}}^{\mathrm{ccs}^{2}}
$$

where

$r_{\max } \quad$ Maximum radius on cones bottom

$h_{\max } \quad$ Maximum cone height

The necessary transformation between local cone coordinate system and project coordinate system is given by

$$
\mathbf{X}_{\mathrm{lsff}, \mathrm{cam}}^{\mathrm{pcs}}=\mathbf{X}_{\mathbf{0}}^{\mathrm{pcs}}+m_{\mathrm{ccs}}^{\mathrm{pcs}} \cdot \mathbf{R}_{\mathrm{ccs}}^{\mathrm{pcs}} \cdot \mathbf{X}_{\mathrm{lsrf}, \mathrm{cam}}^{\mathrm{cs}}
$$

\footnotetext{
where

$\mathbf{X}_{\mathbf{O} \text {,cone }}^{\mathrm{pcs}} \quad$ Cone's origin in project coordinate system

$m_{\text {css }}^{\text {pcs }} \quad$ Scale factor

$\mathbf{R}_{\mathrm{ccs}}^{\mathrm{pcs}} \quad$ Rotation matrix
}

\subsection{Functional Model}

2D LSFR: LSRF directly deliver distance and angular observations as well as intensity information. The task is to automate the detection of the cones in each scan line at each position. An analysis of both intensity and range data is performed to identify all measurement points belonging to a reference primitive (figure $3 \mathrm{c}$ ). In detail, the processing chain consists of the following steps:

1. Calculate the mean of the long-term measurements (figures $3 \mathrm{a}$ and $3 \mathrm{~b}$ ) performed at each position and further smooth the resulting single scan lines by moving average (figure $3 \mathrm{c}$; cyan and black graphs)

2. Apply dynamically derived distance and intensity thresholds for further data containment.

3. Find local maxima to roughly detect the positions of the cones (figure $3 \mathrm{c}$; red crosses).

4. Calculate local extrema of distance gradient curves.

5. All measurement points located between two local extrema belong to a cone if and only if a rough cone position detected in (3) is included (figure 3c; red dotted lines).

6. Remove outliers, for instance caused by multipath effects at surface edges.

The distances measured between LSRF optics and cone surfaces serve as first, the corresponding angles of deflection as second observation type:

$$
\begin{gathered}
D=\sqrt{X_{\mathrm{lsf}}^{\mathrm{lcs}^{2}}+Y_{\mathrm{lsr}}^{\mathrm{lcs} 2}+Z_{\mathrm{lsrf}}^{\mathrm{lcs} 2}}+\Delta D \\
\alpha=\arctan \frac{Y_{\mathrm{lsf}}^{\mathrm{lcs}}}{X_{\mathrm{lsrf}}^{\mathrm{lcs}}}+\Delta \alpha
\end{gathered}
$$

The observation equations 8 and 9 include the unknown distance and angular error-correction parameters $\Delta D$ resp. $\Delta \alpha$ introduced in section 3 .

Further unknowns are the 3D coordinates of each scanned point on the cone surfaces, in a first instance as local sensor coordinates $\mathbf{X}_{\text {lsrf }}^{\mathrm{lcs}}$. Using equation 4 results in higher-level project coordinate $\mathbf{X}_{\mathrm{lsrf}}^{\mathrm{pcs}}$ which have to fulfill the constraint equation 6 including the unknown cone parameters radius $r_{\max }$ and height $h_{\max }$.

The fact that a 2D LSRF measures in one horizontal laser scanner plane only has taken into account by the following constraint which forces the vertical angle $\beta$ to be zero:

$$
\beta=\arctan \frac{Z_{\mathrm{lsrf}}^{\mathrm{lcs}}}{\sqrt{X_{\mathrm{lsrf}}^{\mathrm{lcs} 2}+Y_{\mathrm{lsrf}}^{\mathrm{lsc}^{2}}}}:=0
$$

Supporting DSLR camera: A convergent multi-image set of a DSLR camera is taken of all 3D reference primitives. The positions of circular coded targets have been detected and measured automatically with a standard deviation of $1 / 25$ pixel using the photogrammetric software package AICON $3 D$ Studio (figure 4). These image point coordinates form the third type of observations introduced into the bundle adjustment. The well-known collinearity equations as inverse function of equation 5 are the 


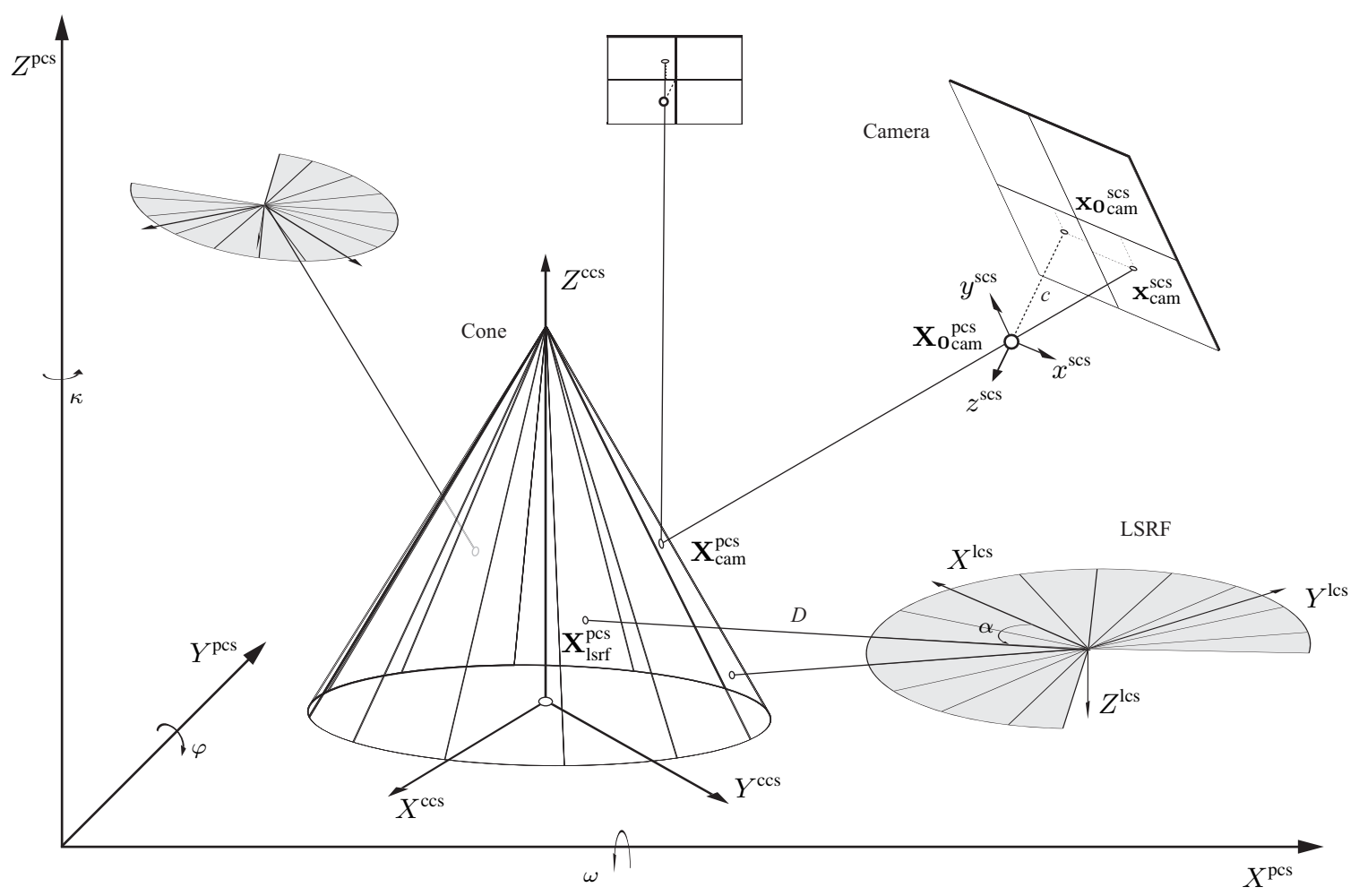

Figure 2: Geometric model.

corresponding observation equations for mapping an object point $\mathbf{X}_{\mathrm{cam}}^{\mathrm{pcs}}(X, Y, Z)$ to an image point $\mathbf{x}_{\mathrm{cam}}^{\mathrm{scs}}(x, y)$ using a central projection:

$$
\begin{aligned}
& x=x_{0} \\
& -c \cdot \frac{r_{11} \cdot\left(X-X_{0}\right)+r_{21} \cdot\left(Y-Y_{0}\right)+r_{31} \cdot\left(Z-Z_{0}\right)}{r_{13} \cdot\left(X-X_{0}\right)+r_{23} \cdot\left(Y-Y_{0}\right)+r_{33} \cdot\left(Z-Z_{0}\right)} \\
& +\Delta x \\
& y=y_{0} \\
& -c \cdot \frac{r_{12} \cdot\left(X-X_{0}\right)+r_{22} \cdot\left(Y-Y_{0}\right)+r_{32} \cdot\left(Z-Z_{0}\right)}{r_{13} \cdot\left(X-X_{0}\right)+r_{23} \cdot\left(Y-Y_{0}\right)+r_{33} \cdot\left(Z-Z_{0}\right)} \\
& +\Delta y
\end{aligned}
$$

$\begin{array}{ll}\text { where } & \\ c & \text { Focal length } \\ \mathbf{x}_{\mathbf{0}} & \text { Principal point } \\ \Delta \mathbf{x} & \text { Correction functions } \\ \mathbf{X}_{\mathbf{0}} & \text { Projection center } \\ r_{r, c} & \text { Elements of a rotation matrix } \mathbf{R}\end{array}$

The unknowns which can be estimated from equation 11 are the focal length $c$, the principal point $\mathbf{x}_{\mathbf{0}}$ and the parameters of the image correction functions $\Delta \mathrm{x}$ as interior orientation parameters as well as the exterior orientation parameters $\mathbf{X}_{\mathbf{0}}{ }_{0 \text { cam }}^{\mathrm{pcs}}$ and $\mathbf{R}_{\mathrm{scs}}^{\mathrm{pcs}}(\omega, \varphi, \kappa)$ of the supporting DSLR camera. Further, the coordinates $\mathbf{X}_{\text {cam }}^{\text {pcs }}(X, Y, Z)$ of all object points signalized on the cone surfaces can be calculated in project coordinate system. Like the $3 \mathrm{D}$ coordinates determined by the LSRF, they should also satisfy the general equation 6 of a cone.

Additional Constraints: A 3D rotation can be described by three Euler angles $(\omega, \varphi, \kappa)$ or, in order to avoid ambiguous trigonometric functions, by four quaternions $\left(q_{1}, q_{2}, q_{3}, q_{4}\right)$. The use of quaternions makes sense from a numerical point of view, but requires one additional equation per LSRF resp. DSLR position

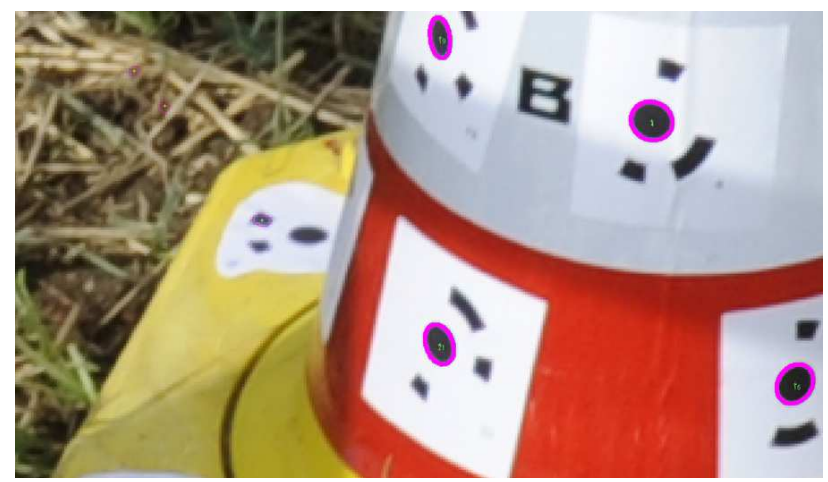

Figure 4: Image point coordinate measurement by ellipse fit.

to enforce an orthogonal rotation matrix $\mathbf{R}$ :

$$
1=q_{1}^{2}+q_{2}^{2}+q_{3}^{2}+q_{4}^{2}
$$

The reference frame of the integrated self-calibrating bundle approach should be adjusted as an unconstrained network. The rank defect of the resulting singular system of equations can be removed by including seven additional constraints: 3 translations, 3 rotations, 1 scaling factor, (e. g. Luhmann et al., 2006). The scale was determined by two diagonal reference distances across the target field and used to fix the scale factors $m^{\text {pcs }}$ within the transformation equations 4,5 and 7 .

\subsection{Stochastic Model}

The stochastic model contains information about the accuracy of the functional model, especially the weighting of the observations and constraints. Usually, information from the instrument manufacturer or from previous accuracy analyses provide the basis for specifying the a-priori variance of a measurement. The integrated calibration method combines heterogeneous observations 


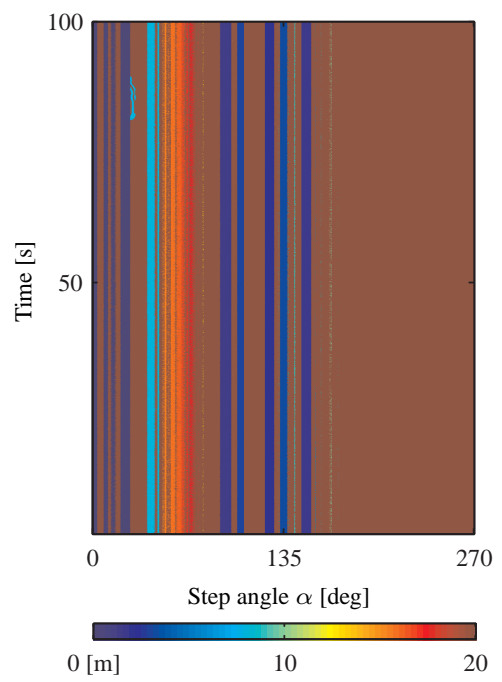

(a)

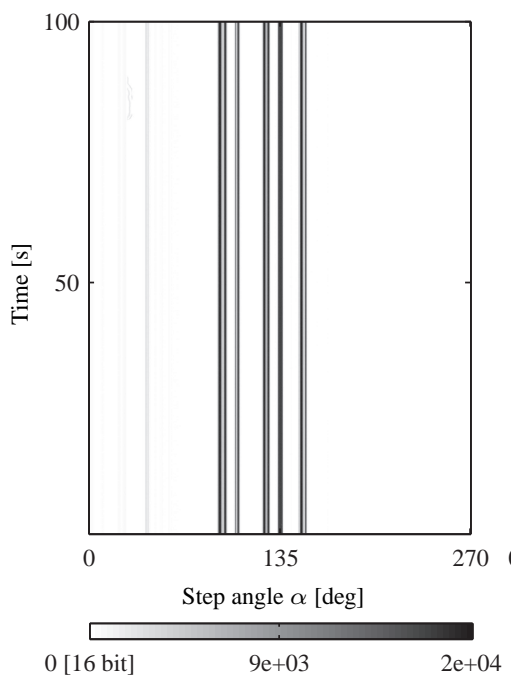

(b)

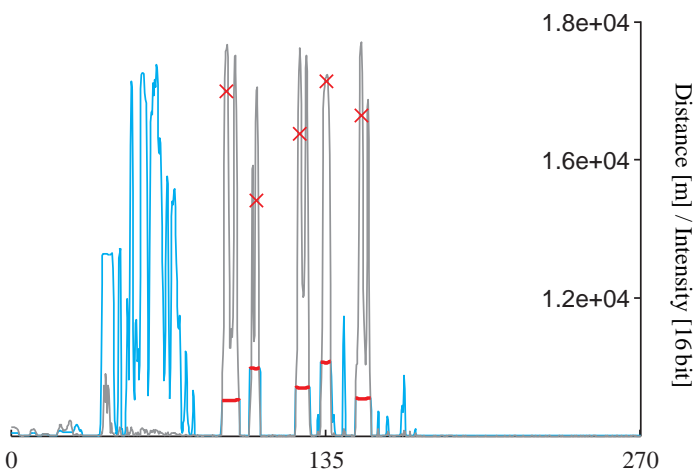

Step angle $\alpha[\mathrm{deg}]$

(c)

Figure 3: Part of (a) range and (b) intensity LSRF raw data captured from one position over time. The five bluish/black lines in range/intensity data (in a distance of approx. $5 \mathrm{~m}$ around the region of $135 \mathrm{deg}$ ) clearly represent the five cones. The result of the segmentation of the cones is shown in (c): The cyan and the black curves represent the mean of the long-term measurements in distance resp. intensity channel. The red crosses roughly mark the detected cone positions, and the red lines highlight the corresponding measurement points which are introduced into the bundle adjustment.

with unknown accuracy as well as different constrained geometric relations. The expansion of the stochastic model to the geodetic concept of include variance component estimation (VCE; Kubik, 1967; Förstner, 1979) ensures that this heterogeneous information pool is fully exploited.

The stochastic model is represented by the variance-covariance matrix $\boldsymbol{\Sigma}_{l l}$ of the observations before the adjustment process. The weights of the observations are given by the quotient of $s_{0}^{2}$ to $s_{i}^{2}$. The variance of the unit weight $s_{0}^{2}$ is a constant, and $s_{i}$ are the variances of the observations, namely the variance component $s_{D}^{2}$ for the LSRF distance measurements, $s_{\alpha}^{2}$ for tapping the LSRF deflection angles and $s_{x y}^{2}$ for the DSLR image point measurements. To differentiate between constant and distance-related error components, the group variance for the distance measurement is further separated into two adaptive variance components $s_{D}^{2}=s_{D 0}^{2}+s_{D 1}^{2} \cdot D^{2}$ (Sieg and Hirsch, 2000). $\boldsymbol{\Sigma}_{l l}$ can now be subdivided into three components, i.e., one (adaptive) component per group of observation:

$$
\begin{aligned}
\boldsymbol{\Sigma}_{l l} & =\operatorname{diag}\left(\boldsymbol{\Sigma}_{D}, \boldsymbol{\Sigma}_{\alpha}, \boldsymbol{\Sigma}_{x y}\right) \\
& =\operatorname{diag}\left(\left(s_{D 0}^{2}+s_{D 1}^{2} \cdot D^{2}\right) \mathbf{I}, s_{\alpha}^{2} \mathbf{I}, s_{x y}^{2} \mathbf{I}\right)
\end{aligned}
$$

where

I Identity matrix

The remaining additional constraints for the non-existent vertical angle (equation 10), the implementation of quaternions (equation 12) as well as for a free network adjustment are considered to be mathematically rigorous by $\mathbf{P}_{C}=\mathbf{0}$ in the extended system of normal equations (Snow, 2002; section 4.4). Solely the geometric cone model is introduced less restrictive due to imperfections in the manufacturing of the traffic cones, which were used as reference primitives (equation 6; section 5.1). Their weights are adjusted automatically as fourth variance component group.

In the course of the VCE, the approximate values for the variance components are improved within a few iterations. See (Koch, 1999) for further information.

\subsection{Solving the Adjusmtent Task}

The integrated bundle adjustment bases on an extended GaussMarkov model:

$$
\begin{gathered}
\mathbf{A} \hat{\mathbf{x}}-\mathbf{l}=\mathbf{v} ; \quad \mathbf{B} \hat{\mathbf{x}}+\mathbf{w}=\mathbf{0} \\
\mathbf{v}^{\mathrm{T}} \mathbf{P} \mathbf{v}+2 \mathbf{k}(\mathbf{B} \hat{\mathbf{x}}+\mathbf{w}) \rightarrow \min \\
{\left[\begin{array}{c}
\hat{\mathbf{x}} \\
\mathbf{k}
\end{array}\right]=\left[\begin{array}{cc}
\mathbf{A}^{\mathrm{T}} \mathbf{P A} & \mathbf{B}^{\mathrm{T}} \\
\mathbf{B} & -\mathbf{P}_{C}^{-1}
\end{array}\right]^{-1}\left[\begin{array}{c}
\mathbf{A}^{\mathrm{T}} \mathbf{P} \mathbf{l} \\
\mathbf{w}
\end{array}\right]}
\end{gathered}
$$

where

v Residuals

k Lagrangian multipliers

The functional model (section 4.2) is required to set up the coefficient matrices $\mathbf{A}$ and $\mathbf{B}$, which contain the linearized observation and constraint equations, the reduced observation vector $\mathbf{l}$ and the vector of inconsistencies $\mathbf{w}$. The weight matrices $\mathbf{P}$ and $\mathbf{P}_{C}$ as inverse variance-covariance matrices $\boldsymbol{\Sigma}_{l l}$ resp. $\boldsymbol{\Sigma}_{C}$ define the stochastic model (section 4.3). The extended system of normal equations is solved iteratively. At each step, the solution vector $\hat{\mathbf{x}}$ is added to the approximate values of the previous iteration until the variances reach a minimum and the optimization criterion is fulfilled.

As a least squares adjustment, the method delivers information on the precision, determinability and reliability of the unknown parameters. This includes the a-posteriori variance $s_{i}^{2}$ of each of the parameters as well as the correlation between parameters. In combination with an automatic VCE, a-posteriori variances $s_{l}^{2}$ and $s_{\hat{l}}^{2}$ of the original resp. adjusted observations can further be stated. Data snooping techniques for gross error detection are implemented as well as a student test to decide whether an introduced parameter is significant or not. The least squares adjustment is repeated until no gross error remains and all used parameters are significant. 


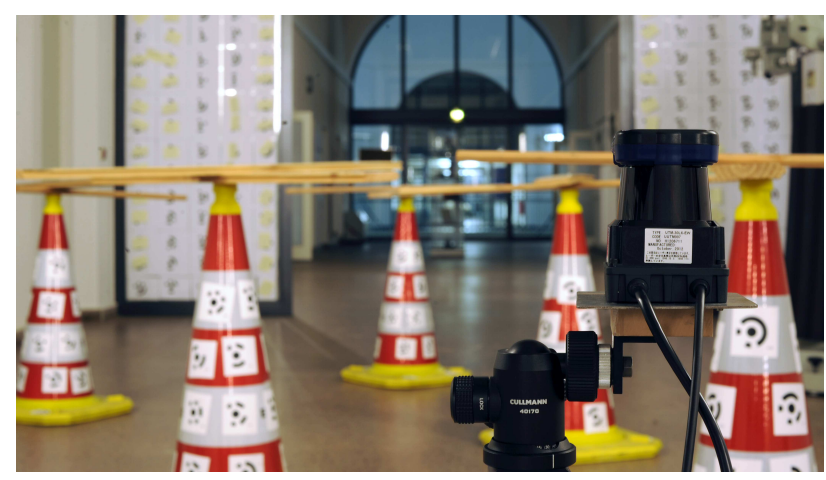

Figure 5: Measuring device Hokuyo UTM-30LX-EW in front of the target field.

\section{RESULTS}

\subsection{Experimental Configuration}

A 3D calibration target field was designed in order to proof the concept presented here (figure 5). It consists of eight standard retro-reflective traffic cones with a maximum height $h_{\max }$ of $90 \mathrm{~cm}$ and a maximum radius $r_{\max }$ of $15 \mathrm{~cm}$. The cones are spatial distributed over an area of approximately $10 \mathrm{~m} \times 5 \mathrm{~m}$. Six LSRF positions in distances of up to $15 \mathrm{~m}$ allow for the determination of all distance- and angular-related correction terms. LSRF scans in larger distances could not be oriented reliably. The 3D target field was further captured by a supporting DSLR camera. Overall 100 convergent images, some of them rolled against the camera axis, ensure a stable network geometry.

\subsection{LSRF Calibration Parameters}

Table 1 lists the LSRF calibration parameters and the corresponding a-posteriori standard deviations estimated within the integrated bundle adjustment. The additive term $a_{0}$ of the distance correction model $\Delta D$ is $9 \mathrm{~mm}$, the multiplicative parameter $a_{1}$ amounts to $0.19 \%$ of the measured distance. The circle scale $b_{1}$ to correct errors in horizontal direction is $-0.21 \%$ of the deflection to the zero point. The eccentricity of the collimation axis to the rotation axis causes a correction of up to $3.5 \mathrm{~mm}$. Further parameters of the correction model $\Delta \alpha$ for errors in horizontal direction could not be estimated significantly.

Considerable correlations between the LSRF calibration terms could not be observed. The highest coefficient is about 0.7 between the distance correction parameters $a_{0}$ and $a_{1}$.

\subsection{LSRF Pose}

The pose of a single LSRF view point can be stated with an aposteriori RMS of $1.7 \mathrm{~mm}$ resp. $7.2 \mathrm{~mm}$ for the position of the projection center in $x y$ - resp. $z$-direction of the project coordinate system. The mean RMS deviation of the rotation components is $0.14 \mathrm{deg}$. The uncertainties in determining LSRF height components can be explained by the unfavorable ratio between the maximum radius and the height of the traffic cones used.

\subsection{Cone Parameters and Pose}

The parameters as well as the poses of the cone primitives are estimated by directional LSRF distance measurements and DSLR image coordinate measurements. The unknown cone parameters are calculated with a RMS error of $0.5 \mathrm{~mm}$ for a maximum radius of $14.34 \mathrm{~cm}$ and $8.8 \mathrm{~mm}$ for a height of $90.13 \mathrm{~cm}$, and the poses with a mean a-posteriori standard deviation of $0.11 \mathrm{~mm}$ for the shift and $9.46 \mathrm{e}^{-3} \mathrm{deg}$ for the rotation.

\subsection{D Object Coordinates}

The mean a-posteriori standard deviation of the cone surface points $\mathbf{X}_{\text {lsf }}$ observed by the LSRF is $4.8 \mathrm{~mm}$ in lateral direction and $9.2 \mathrm{~mm}$ in height. The precision of a 3D object point $\mathbf{X}_{\text {cam }}$ estimated from DSLR image coordinate measurements is $0.09 \mathrm{~mm}$.

\subsection{Residuals}

The residuals $v_{D}$ of the distance measurements are shown in figure 7 for an adjustment with and without considering the LSRF error correction model. The red graph results from of a function fitted into the residuals of the uncorrected distance measurements. It indicates a slight constant offset and a distance-related trend. The RMS of the residuals is $0.63 \mathrm{~mm}$, the expected value $\mu$ is $-0.28 \mathrm{~mm}$. The black graph of a function fitted into the remaining residuals after a adjustment parametrized with LSRF correction terms is nearly a straight line with $y=0=$ const. The normally distributed residuals do not show interpretable effects. The RMS is equal to the previous solution, but the expected value $\mu=0.8 \mu \mathrm{m}$ tends more clearly towards zero.

The situation is similar for the residuals of the angular measurements: The RMS of the angular residuals of an un-parametrized estimation is $0.1 \mathrm{deg}\left(\mu=2.52 \mathrm{e}^{-2} \mathrm{deg}\right)$. If calibration parameters are taken into account, the RMS is reduced to $0.06 \mathrm{deg}$ $\left(\mu=9.05 \mathrm{e}^{-3} \mathrm{deg}\right)$.

The normally distributed residuals $v_{x y}$ of the image coordinate measurements do not show any systematic effect. They vary in both coordinate directions with an a-posteriori RMS deviation $s_{v x y}$ about $1 / 20$ pixel around the expected value $\mu=1.7 \mathrm{e}^{-3} \mu \mathrm{m}$.

Even though probably not all LSRF effects are considered, the results show that the integration of LSRF correction parameters is better suited to model the geometric-physical reality of a LSRF measurement process.

\subsection{Observational Errors}

The a-posteriori standard deviations of the original observations estimated automatically by VCE as well as of the adjusted observations calculated in the course of the error analysis after the bundle adjustment are shown in table 2 .

\begin{tabular}{|c|c|c|c|}
\hline$\hat{s}_{D 0}$ & $\hat{s}_{D 1}$ & $\hat{s}_{\alpha}$ & $\hat{s}_{x y}$ \\
\hline not significant & $1.95 \mathrm{e}^{-3}$ & $3.46 \mathrm{e}^{-3} \mathrm{rad}$ & $0.57 \mu \mathrm{m}$ \\
\hline \hline \multicolumn{2}{|c|}{$\hat{s}_{\hat{D}}$} & $\hat{s}_{\hat{\alpha}}$ & $\hat{s}_{\hat{x} \hat{y}}$ \\
\hline \multicolumn{2}{|c|}{$1.47 \mathrm{~mm}$} & $2.39 \mathrm{e}^{-3} \mathrm{rad}$ & $0.19 \mu \mathrm{m}$ \\
\hline
\end{tabular}

Table 2: A-posteriori standard deviations $\hat{s}$ of the original and the adjusted observations.

The deviation $\hat{s}_{D}$ of an original LSRF distance measurement is about $0.20 \%$ of the measured distance $D$. Depending on the measuring range $[1,15 \mathrm{~m}]$ specified in section 5.1 , the accuracy can thus be stated with $[2.0,30 \mathrm{~mm}]$. These values correspond to the level of precision specified by the manufacturer (Hokuyo, 2014). Remark that the constant offset $\hat{s}_{D 0}^{2}$ as one of the two adaptive variance components could not be estimated reliably. It was excluded from the variance component estimation process. At first sight this seems exceptional for time-of-flight ranging system, since distance measurements are primarily limited by the precision of the time measurement. The contradiction can 


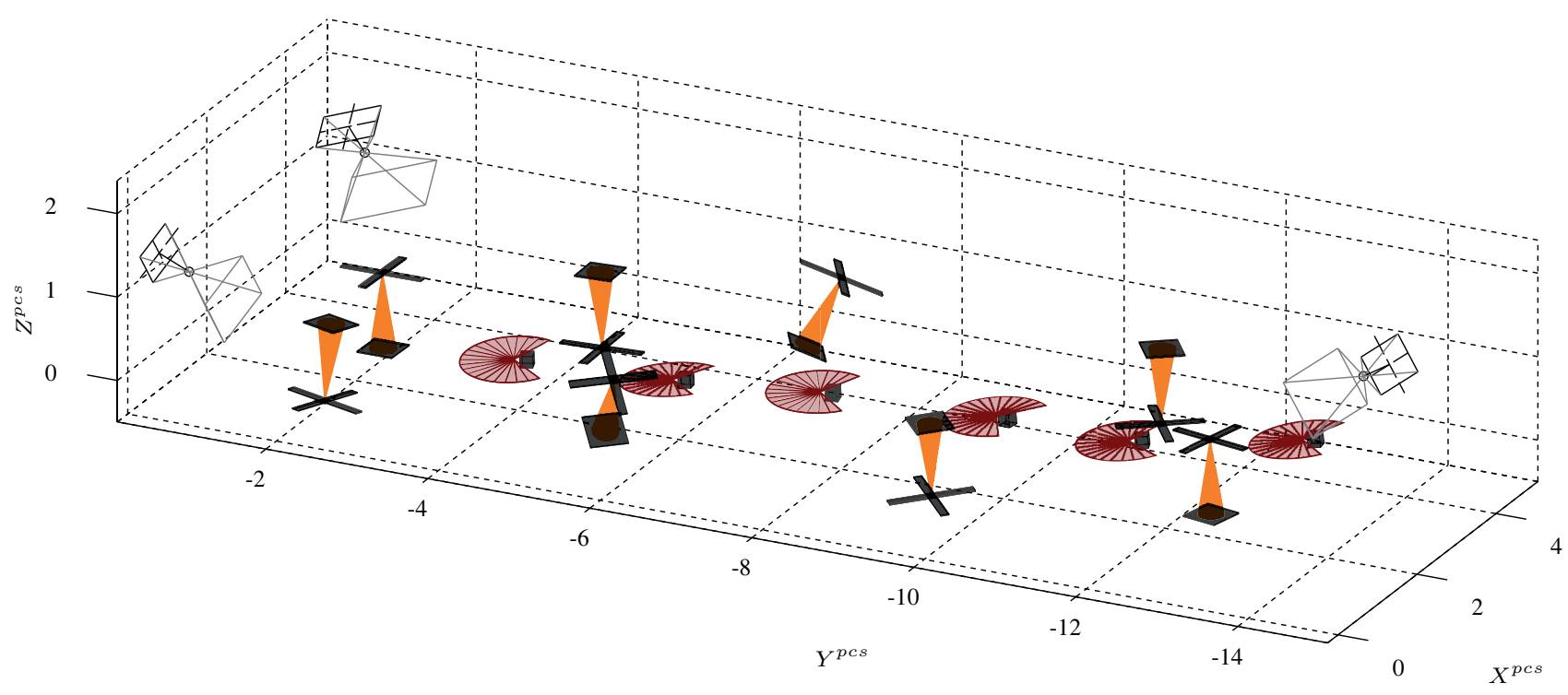

Figure 6: Network geometry: All eight traffic cones and six LSRF positions (represented as fans) are shown, but only a few camera poses for reasons of clarity. The crosses on top as well as the different orientations of the cones additionally ensure a stable network geometry.

\begin{tabular}{|c|c|c|c|c|c|c|c|c|}
\hline & $\begin{array}{c}a_{0} \\
(\mathrm{~mm})\end{array}$ & $a_{1}$ & $b_{1}$ & $b_{2}$ & $b_{3}$ & $b_{4}$ & $b_{5}$ & $\begin{array}{c}b_{6} \\
(\mathrm{~mm})\end{array}$ \\
\hline$\hat{x}_{i}$ & 8.62 & $1.89 \mathrm{e}^{-3}$ & $-2.10 \mathrm{e}^{-3}$ & 0 & 0 & 0 & 0 & 3.32 \\
\hline$\hat{s}_{\hat{x}_{i}}$ & 1.09 & $3.54 \mathrm{e}^{-4}$ & $3.87 \mathrm{e}^{-4}$ & fix & fix & fix & fix & 1.64 \\
\hline
\end{tabular}

Table 1: LSRF calibration parameters $\hat{x}_{i}$ with their standard deviations $\hat{s}_{\hat{x}_{i}}$.

only be explained by an excessive distance-related influence of the beam divergence on the measurement precision of a Hokuyo UTM-30LX-EW (for more detail see paragraph below).

The deflection angle $\alpha$ is specified with an a-priori standard deviation of $0.2 \mathrm{deg}$, which corresponds to $3 / 4$ of the angular step width. This order of magnitude seems to be quite high. One reason for this might be found in the beam divergence. According to manufactures information, the elliptical laser spot size of the Hokuyo UTM-30LX-EW is $50 \mathrm{~mm} \times 500 \mathrm{~mm}$ at sensor's maximum distance of $30 \mathrm{~m}$. This footprint corresponds to an angular widening of approximately $0.1 \mathrm{deg} \times 1.0 \mathrm{deg}$. Further, the orientation of the laser spot (in scan direction resp. across scan direction) depends on the deflection angle and alternates within a single scan line.

In average, the a-posteriori standard deviation $\hat{s}_{x y}$ of an image coordinate measurement is $1 / 15$ pixel. It is thus slightly worse than the precision of the pure image point measurement by ellipse fit as stated in section 4.2 .

The a-posteriori standard deviation $\hat{s}_{0}$ of the unit weight is near to the a-priori constant value $s_{0}=100$, which indicates an optimally determined accuracy ratio for all groups of observations. This implies that the a-posteriori variances of the original observations are equal to their a-priori variances.

The mean standard deviations $\hat{s}_{\hat{D}}$ and $\hat{s}_{\hat{\alpha}}$ of the adjusted LSRF observations are $1.5 \mathrm{~mm}$ for the distance measurement and $0.1 \mathrm{deg}$ for the angular values. In average, the a-posteriori standard deviation $\hat{s}_{\hat{x} \hat{y}}$ of an image coordinate measurement becomes $1 / 43$ pixel.

\section{CONCLUSION AND OUTLOOK}

The flexible self-calibrating bundle adjustment approach presented in this contribution determines a geometric correction model of a 2D single-layer LSRF and estimates all distance- and angular-related correction parameters. The heterogeneous information pool is fully exploited by estimating variance components automatically within the integrated stochastic model. The experimental configuration of the self-calibration is based on a portable 3D target field, whose geometry is determined simultaneously in the adjustment. Complex experimental set-ups can thus be avoided.

The process validation showed that the integration of LSRF calibration parameters leads to a more accurate solution. The accuracy of an original LSRF range measurement can be stated with approximately $0.2 \%$ of the measured distance and with $0.2 \mathrm{deg}$ for the angle specifications. The RMS error of a 3D coordinate after the calibration becomes $5 \mathrm{~mm}$ in lateral and $9 \mathrm{~mm}$ in depth direction.

Future work can concentrate on an improved experimental setup with wide-angle $3 \mathrm{D}$ cone primitives to improve the network geometry. The influence of the beam divergence as well as the dependencies of the LSRF distance measurements on reflectance due to different surface properties should be analyzed. As soon as effects of remaining error sources are investigated, the parametrization of the error correction model can be easily adapted. 


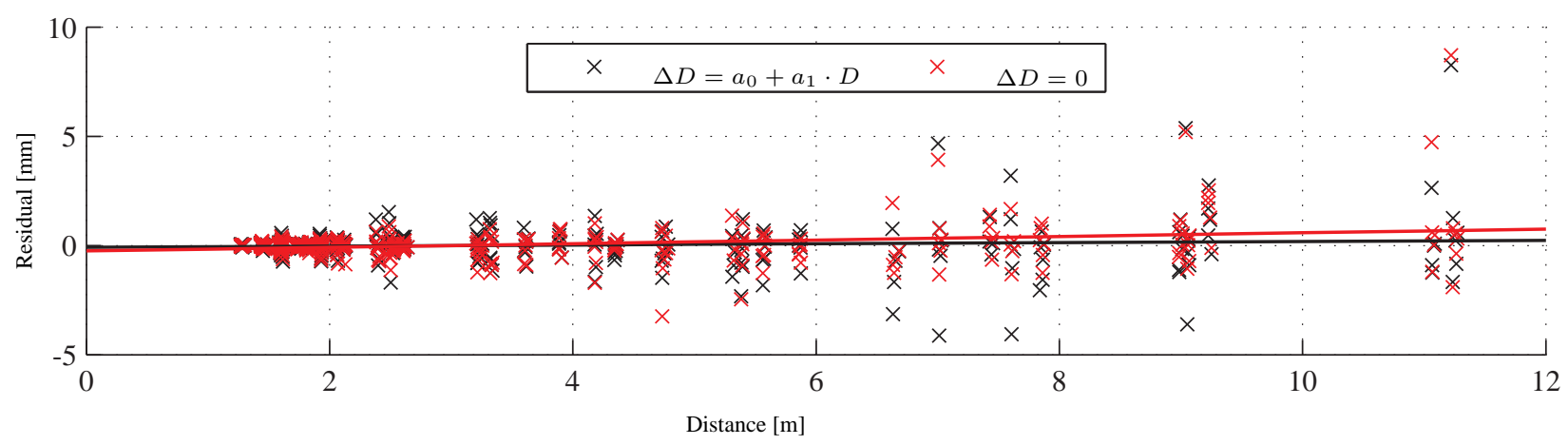

Figure 7: Residuals of the distance measurements with (black) and without (red) correction parameters.

\section{ACKNOWLEDGEMENTS}

The research work presented in this paper has been funded by the European Social Fund (ESF) via Sächsische Aufbaubank (SAB).

\section{References}

Antone, M. E. and Friedman, Y., 2007. Fully automated laser range calibration. In: BMVC, British Machine Vision Association.

Djuricic, A. and Jutzi, B., 2013. Supporting uavs in low visibility conditions by multiple-pulse laser scanning devices. In: C. Heipke, K. Jacobsen, F. Rottensteiner and U. Sörgel (eds), High-resolution earth imaging for geospatial information. International Archives of Photogrammetry, Remote Sensing and Spatial Information Sciences, Vol. XL-1/W1, pp. 93-98.

Förstner, W., 1979. Ein Verfahren zur Schätzung von Varianz- und Kovarianzkomponenten. Allgemeine Vermessungsnachrichten 86, pp. 41-49.

Georgiev, A. and Allen, P. K., 2004. Localization methods for a mobile robot in urban environments. IEEE Transactions on Robotics 20, pp. 851-864.

Gielsdorf, F., Rietdorf, A. and Gruendig, L., 2004. A concept for the calibration of terrestrial laser scanners. In: Proc. of FIG Working Week, Athens, Greece.

Glennie, C. and Lichti, D. D., 2010. Static calibration and analysis of the velodyne hdl-64e s2 for high accuracy mobile scanning. Remote Sensing 2(6), pp. 1610-1624.

Hokuyo, 2014. Product Information UTM-30LX-EW. Hokuyo Automatic Co., Ltd., Japan.

Holz, D., Nieuwenhuisen, M., Droeschel, D., Schreiber, M. and Behnke, S., 2013. Towards multimodal omnidirectional obstacle detection for autonomous unmanned aerial vehicles. ISPRS - International Archives of the Photogrammetry, Remote Sensing and Spatial Information Sciences XL-1/W2, pp. 201-206.

Jain, S., Nandy, S., Chakraborty, G., Kumar, C., Ray, R. and Shome, S., 2011. Error modeling of laser range finder for robotic application using time domain technique. In: IEEE International Conference on Signal Processing, Communications and Computing (ICSPCC), pp. $1-5$.

Kim, J.-B. and Kim, B.-K., 2011. Efficient calibration of infrared range finder pbs-03jn with scan-wise cubic hermite splines for indoor mobile robots. In: 8th International Conference on Ubiquitous Robots and Ambient Intelligence (URAI), IEEE, pp. 353-358.
Kneip, L., Tâche, F., Caprari, G. and Siegwart, R., 2009. Characterization of the compact hokuyo urg-04lx $2 \mathrm{~d}$ laser range scanner. In: IEEE International Conference on Robotics and $\mathrm{Au}-$ tomation, IEEE, pp. 1447-1454.

Koch, K.-R., 1999. Parameter estimation and hypothesis testing in linear models. Springer.

Krüger, T., Nowak, S., Matthaei, J. and Bestmann, U., 2013. Single-layer laser scanner for detection and localization of unmanned swarm members. ISPRS - International Archives of the Photogrammetry, Remote Sensing and Spatial Information Sciences XL-1/W2, pp. 229-234.

Kubik, K., 1967. Schätzung der Gewichte der Fehlergleichungen beim Ausgleichungsproblem nach vermittelnden Beobachtungen. Zeitschrift für Vermessungswesen 92, pp. 173-178.

Kuhnert, K.-D. and Kuhnert, L., 2013. Light-weight sensor package for precision $3 \mathrm{~d}$ measurement with micro uavs e.g. powerline monitoring. ISPRS - International Archives of the Photogrammetry, Remote Sensing and Spatial Information Sciences XL-1/W2, pp. 235-240.

Lichti, D. D., 2007. Error modelling, calibration and analysis of an am-cw terrestrial laser scanner system. \{ISPRS Journal of Photogrammetry and Remote Sensing 61(5), pp. 307 - 324.

Lichti, D. D., 2009. Terrestrial laser scanner self-calibration: Correlation sources and their mitigation. ISPRS Journal of Photogrammetry and Remote Sensing 65(1), pp. 93-102.

Lichti, D. D. and Gordon, S. J., 2004. Error propagation in directly georeferenced terrestrial laser scanner point clouds for cultural heritage recording. In: Proc. of FIG Working Week, Athens, Greece.

Luhmann, T., Robson, S., Kyle, S. and Harley, I., 2006. Close Range Photogrammetry: Principles, Methods and Applications. Revised edition edn, Whittles Publishing.

Maier, D. and Kleiner, A., 2010. Improved gps sensor model for mobile robots in urban terrain. In: ICRA, IEEE, pp. 43854390.

Nowak, S., Krüger, T., Matthaei, J. and Bestmann, U., 2013. Martian swarm exploration and mapping using laser slam. ISPRS - International Archives of the Photogrammetry, Remote Sensing and Spatial Information Sciences XL-1/W2, pp. 299-303.

Okubo, Y., Ye, C. and Borenstein, J., 2009. Characterization of the hokuyo urg-04lx laser rangefinder for mobile robot obstacle negotiation. In: G. R. Gerhart, D. W. Gage and C. M. Shoemaker (eds), Unmanned Systems Technology XI, Proc. of SPIE, Vol. 7332. 
Rogers III, J. G., Trevor, A. J., Nieto-Granda, C., Cunningham, A., Paluri, M., Michael, N., Dellaert, F., Christensen, H. I. and Kumar, V., 2010. Effects of sensory precision on mobile robot localization and mapping. In: Experimental Robotics, Springer, pp. 433-446.

Scherer, S., Rehder, J., Achar, S., Cover, H., Chambers, A. D., Nuske, S. T. and Singh, S., 2012. River mapping from a flying robot: state estimation, river detection, and obstacle mapping. Autonomous Robots 32(5), pp. $1-26$.

Schneider, D. and Maas, H.-G., 2007. Integrated bundle adjustment of terrestrial laser scanner data and image data with variance component estimation. The Photogrammetric Journal of Finland 20, pp. 5-15.

Schneider, D. and Schwalbe, E., 2008. Integrated processing of terrestrial laser scanner data and fisheye-camera image data. International Archives of Photogrammetry, Remote Sensing and Spatial Information Science 37, pp. 1037-1044.

Serranoa, D., Uijt de Haag, M., Dill, E., Vilardaga, S. and Duan, P., 2014. Seamless indoor-outdoor navigation for unmanned multi-sensor aerial platforms. ISPRS - International Archives of the Photogrammetry, Remote Sensing and Spatial Information Sciences XL-3/W1, pp. 115-122.

Sieg, D. and Hirsch, M., 2000. Varianzkomponentenschätzung in ingenieurgeodätsichen Netzen, Teil 1: Theorie. Allgemeine Vermessungsnachrichten 3, pp. 82-90.

Snow, K. B., 2002. Applications of Parameter Estimation and Hypothesis Testing of GPS Network Adjustments. Technical Report 465, Geodetic and GeoInformation Science, Department of Civil Environmental Engineering and Geodetic Science, The Ohio State University, Columbus, Ohio, USA.

Vosselman, G. and Maas, H.-G. (eds), 2010. Airborne and Terrestrial Laser Scanning. Whittles Publishing, Caithness, UK.

Westfeld, P. and Maas, H.-G., 2013. Integrated 3d range camera self-calibration. PFG Photogrammetrie, Fernerkundung, Geoinformation 2013(6), pp. 589-602.

Ye, C. and Borenstein, J., 2002. Characterization of a 2-d laser scanner for mobile robot obstacle negotiation. In: In Proceedings of the IEEE International Conference on Robotics and Automation, pp. 2512-2518. 